\title{
OPEN Determination of critical decision points for COVID-19 measures in Japan
}

\author{
Junu Kim ${ }^{1,2 \bowtie}$, Kensaku Matsunami ${ }^{1,2}$, Kozue Okamura ${ }^{1,2}$, Sara Badr ${ }^{1}$ \& Hirokazu Sugiyama ${ }^{1}$
}

Coronavirus disease 2019 (COVID-19) has spread throughout the world. The prediction of the number of cases has become essential to governments' ability to define policies and take countermeasures in advance. The numbers of cases have been estimated using compartment models of infectious diseases such as the susceptible-infected-removed (SIR) model and its derived models. However, the required use of hypothetical future values for parameters, such as the effective reproduction number or infection rate, increases the uncertainty of the prediction results. Here, we describe our model for forecasting future COVID-19 cases based on observed data by considering the time delay $\left(t_{\text {delay }}\right)$. We used machine learning to estimate the future infection rate based on real-time mobility, temperature, and relative humidity. We then used this calculation with the susceptible-exposedinfectious-removed (SEIR) model to forecast future cases with less uncertainty. The results suggest that changes in mobility affect observed infection rates with 5-10 days of time delay. This window should be accounted for in the decision-making phase especially during periods with predicted infection surges. Our prediction model helps governments and medical institutions to take targeted early countermeasures at critical decision points regarding mobility to avoid significant levels of infection rise.

Coronavirus disease 2019 (COVID-19) has spread throughout the world ${ }^{1}$. Limited information about the COVID-19 pandemic leaves governments no choice but to take reactive measures ${ }^{2-4}$. In Japan, states of emergency were declared on 7 April 2020 and 8 January 2021, but both occurred after the number of cases had increased significantly. Knowing the expected evolution of infection rates and the magnitude of potential increases beforehand would help governments to initiate countermeasures at an appropriate decision point to balance the economic and social consequences of the taken measures. These proactive countermeasures should greatly suppress the increasing spread ${ }^{5}$.

Accurate models for the prediction of potential COVID-19 spread are crucial for timely intervention measures. A review of developed mathematical epidemiological models is given in Allen et al. ${ }^{6}$. Predictions using mathematical models of infectious diseases, such as the SIR and SEIR models, are widely used at present ${ }^{7-17}$. Wang et al. ${ }^{13}$ used the SIR model for forecasting the impacts of intervention methods on the number of cases. Kuniya $^{3}$ evaluated the impact of the first states of emergency in Japan by calculating reproduction numbers from past data. Bubar et al. ${ }^{15}$ have proposed a COVID-19 vaccine prioritization strategy through an age-stratified SEIR model. The difference of SIR and SEIR models is whether the model includes "E", the number of exposed people. This term enables the consideration of the impact of the contact between people on the infection rates. However, these models were fitted to specific time periods $s^{7,10-16}$ and heavily rely on the assumption of complex factors that could be a function of a combination of multiple underlying conditions and policies. For example, the number of infected people is calculated under the assumption of certain values of the effective reproduction number, which can result from the combination of several factors including the implemented policies and general environmental factors such as the weather. Using such an approach for setting effective future policies would imply a dependence on the occurrence of specific scenarios under static conditions.

Critical factors have been identified to affect the number of cases such as mobility ${ }^{18}$ and temperature ${ }^{19-21}$. These factors are dynamic in nature in addition to the evolving situation with the virus itself such as changing variants and vaccination rates. Due to this dynamic nature of the situation, a model that avoids constant assumptions is beneficial. Other approaches such as the use of machine learning and deep learning techniques have been used to model the spread of COVID-1922,23. Although these models show good performance, the target period

${ }^{1}$ Department of Chemical System Engineering, The University of Tokyo, 7-3-1 Hongo, Bunkyo-ku, Tokyo 113-8656, Japan. ${ }^{2}$ These authors contributed equally: Junu Kim, Kensaku Matsunami and Kozue Okamura. ${ }^{\square}$ email: j-kim@ pse.t.u-tokyo.ac.jp 


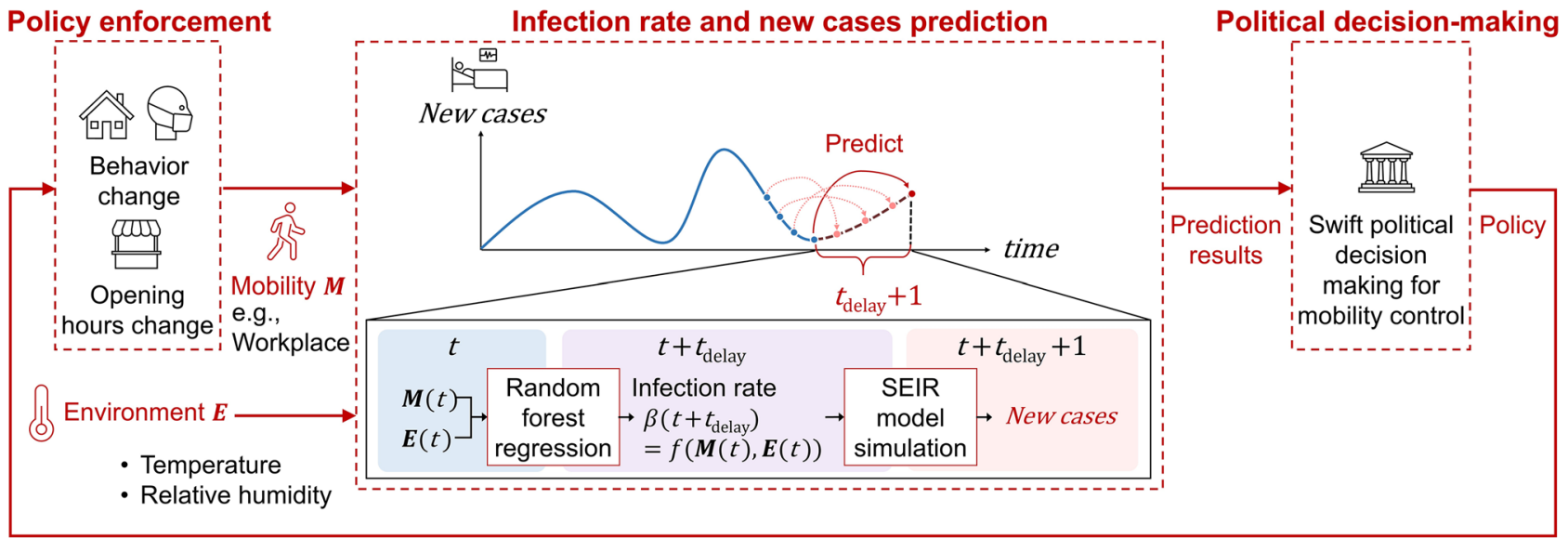

Figure 1. Representation of our modelling approach and predictions. The model comprises two parts. The first part is observed infection rate $\beta(t)$ forecasting. The infection rate $\beta(t)$ is predicted using mobility, temperature, and relative humidity as training data and inputs to the random forest regression. Assuming a time delay between the input measurement day and the day of the change in infection rate $\beta(t)$, the model can be used to forecast the appearance of new COVID-19 cases. The second part is new case forecasting. The number of new cases can be simulated using the predicted infection rate $\beta(t)$ as the input parameter in the SEIR model. The forecast is based on the observed data, and there is no need to assume a change in mobility in the future. Policy decisions will be reflected in changes in behaviour and opening hours, which affect mobility, and will be incorporated into the model with daily updates.

tends to be short ${ }^{22}$. Also, these models can suffer from vulnerability to several shortcomings such as bias in the training data and lack of generalisability ${ }^{23,24}$. Therefore, in order to utilize the advantages of both mathematical modelling and machine learning techniques, this work has extended the SEIR model with a machine learning approach to forecast infection rate based on real-time mobility and weather data.

To account for the future impact of real-time changes, a time lag was assumed. We incorporated the factor $t_{\text {delay }}$ for mobility and environmental factors into our model (Fig. 1). We defined $t_{\text {delay }}$ as the time for mobility and environmental factors to affect the infection rate. Because mobility and environmental factors have a crucial impact on the spread of an epidemic ${ }^{5,8,10,18-21}$, we used machine learning, random forest ${ }^{25}$, to estimate the infection rate of the SEIR model based on these factors. We first directly calculated the infection rate from the COVID-19 data in Japan ${ }^{26-29}$ and then used random forest regression to estimate the infection rate based on different categories of mobility ${ }^{30}$, temperature ${ }^{31}$, and relative humidity ${ }^{31}$. In the process, we found out the impact of mobility and environmental factors on the infection rate is most visible after 5-10 days of $t_{\text {delay }}$. Our approach, which considered this $t_{\text {delay }}$ allowed us to forecast future COVID-19 cases based on the observed data, which enables proactive countermeasures at critical decision points. The model learns new behaviour and is updated in real-time as the training set evolves. The detailed analysis of the impact of each factor on the infection rate provided by this model may be useful for informing policy decisions to tackle the pandemic by controlling one or more specific elements of mobility.

\section{Results}

Random forest regression to estimate infection rate. As described in the "Methods" section, we first calculated the actual daily observed infection rate $\beta(t)$ using the data for COVID-19 cases in Japan. We then used random forest regression to estimate the $\beta(t)$ based on mobility, temperature, and relative humidity. A sensitivity analysis was carried out to determine appropriate values of $t_{\text {delay }}$. We varied the $t_{\text {delay }}$ between 0 and 19 days and compared the resulting coefficients of determination of the actual and estimated $\beta(t)$ values (Supplementary Fig. S1). The range of the $t_{\text {delay }}$ was chosen to reflect the sum of incubation period and additional buffer time until test results were obtained. For COVID-19, previous research suggests the incubation period is around 5 days but can be variable from 1 to 14 days $^{32}$. A $t_{\text {delay }}$ of 6 days showed the best results closely followed by a $t_{\text {delay }}$ of 5 days. The coefficients of determination remained similarly high in the range between 5 and 10 days. This shows that the impact of mobility factors on calculated infection rates can be clearly observed within a window of 5-10 days. This result is reasonable for Japan, where people are allowed to take a PCR (Polymerase Chain Reaction) test after they have shown symptoms or have been in close contact with someone who has tested positive. As symptoms generally manifest 5-6 days after the actual infection ${ }^{33,34}$, it is therefore reasonable that a $t_{\text {delay }}$ of 5-10 days well describes the impact of mobility on observed infection rates. Considering all aspects including the nature of COVID-19 and the use of the model to determine critical decision points, we focused mainly on the model with a 6-day $t_{\text {delay }}$ in the analysis presented below. The same approach to estimate $\beta(t)$ was applied for four prefectures: Tokyo, Osaka, Hokkaido, and Fukuoka.

Forecast results in Japan. The forecast results of the total new COVID-19 cases in Japan for the period (16 October 2020 to 11 February 2021) are presented in Fig. 2. The forecast is obtained using the SEIR model 


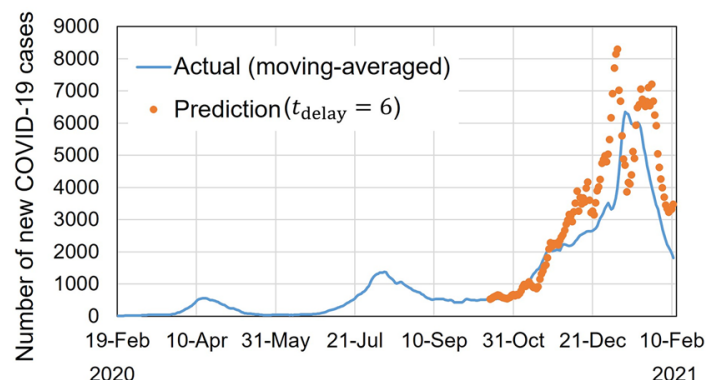

(a)

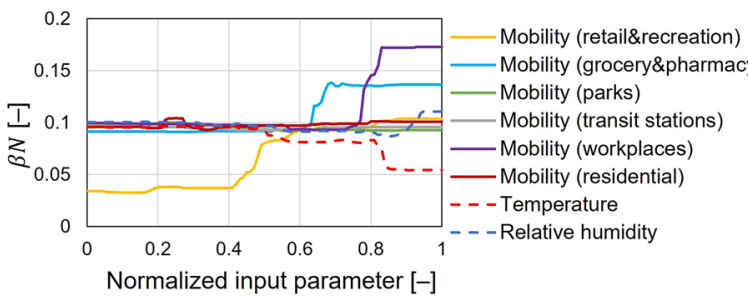

(f)

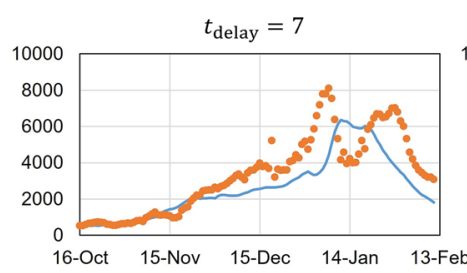

(b)

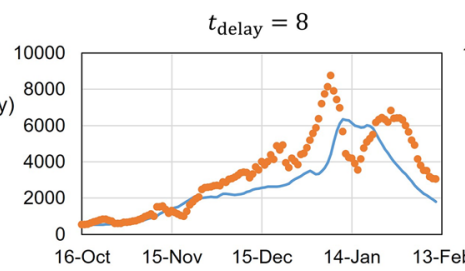

(c)

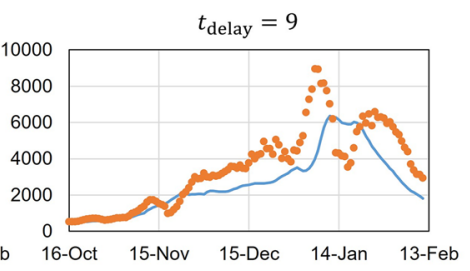

(d)

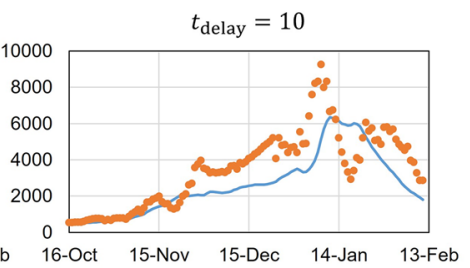

(e)

Figure 2. Forecast and analysis results of the number of COVID-19 cases in Japan. Forecast results using a $t_{\text {delay }}$ of (a) 6 days, (b) 7 days, (c) 8 days, (d) 9 days, (e) 10 days. Forecasting started on 16 October 2020 using the data from 21 February to 15 October 2020 for model development. (f) Sensitivity analysis to quantify the impacts of mobility, temperature, and humidity on the infection rate $\beta$. The random forest model used was developed using the simple moving average data for mobility, temperature, and humidity from 21 February 2020 to 11 February 2021 as training data. Each input parameter was changed from the minimum to the maximum of the training data, and the other input parameters were fixed as the mean values for the training data. The horizontal axis in the graph is the normalized value of input parameters, where the minimum and the maximum were normalized as 0 and 1, respectively. The vertical axis represents the product of the infection rate $\beta$ and population $N$.

with the predicted $\beta(t)$ as an input. The prediction of $\beta(t)$ started from 16 October using mobility data from 6 days ahead (10 October). The predicted infection rates on a certain day $\beta(t)$ was used to forecast the number of cases on the next day at $(t+1)$. The initial training set (from 15 February to 15 October 2020) was updated daily afterwards for further predictions of $\beta(t)$, thus enabling forecasts of $t_{\text {delay }}+1$ day-ahead. Comparing the forecast results using different values of $t_{\text {delay }}$ up to 10 days showed that the prediction could follow the general trend of the number of new cases (Fig. 2), but with forecast errors becoming larger with increasing $t_{\text {delay }}$ especially around the peak of the third wave. Predicted case numbers using models with $t_{\text {delay }}$ of 6-10 days showed the start of the third wave in November 2020. Prediction of the magnitude and timing of the peak varied between the models. Forecasted peak values were slightly higher than the actual (between 27 and $46 \%$ for $t_{\text {delay }}$ of 6-10 days). On the other hand, the timing of both the peak and the start of the decrease in the number of cases was more accurately predicted (3-5 days difference between the actual and predicted peak times for $t_{\text {delay }}$ of 6-10 days).

Figure $2 \mathrm{f}$ shows the impact of different input parameters on the general infection rate $\beta$ throughout the entire period (not time-specific). The parameters with the highest influence were determined as: mobility in retail and recreation, in grocery shops and pharmacies, and in workplaces, in addition to the temperature. Gaps between prediction and actual recorded cases around the third wave result from multiple factors including, changing behaviour from the previous training set with the onset of a new season (lower temperatures in December and January than the training set, which starts in February) or special holidays around the new year which shifted patterns of mobility (Supplementary Fig. S2) and changes in the number of conducted tests.

Forecasts in the four prefectures. We applied the same method to the data from the four prefectures: Tokyo, Osaka, Hokkaido, and Fukuoka, which were selected because of their diversity in geography and climate, population, and the number of COVID-19 cases. Tokyo and Osaka have a higher population density than Hokkaido and Fukuoka. Hokkaido has a different climate being further up north with much colder winters, and milder summer temperatures. The forecast results with different $t_{\text {delay }}$ values 6 days and (7-10 days) are presented in Fig. 3 and Supplementary Fig. S3, respectively.

The model could predict the number of cases in all four prefectures despite the variation in climate, population, and the number of COVID-19 cases (Fig. 3). This suggests that the critical underlying factors have been accounted for in the model, and its application could thus be extended to other areas. The sensitivity of the infection rate to changes in mobility patterns, temperature, and humidity for the four prefectures is presented in Supplementary Fig. S4. 


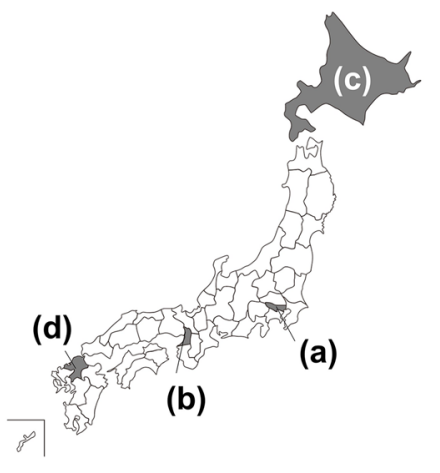

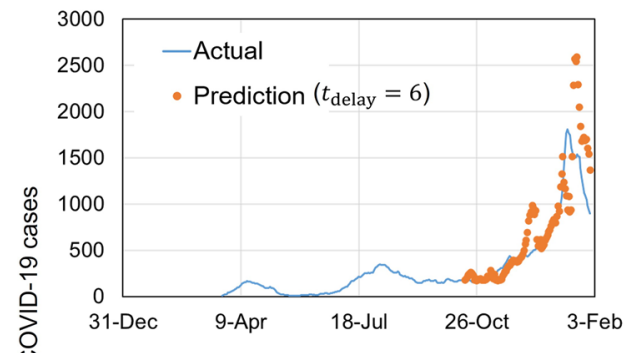

(a) Tokyo

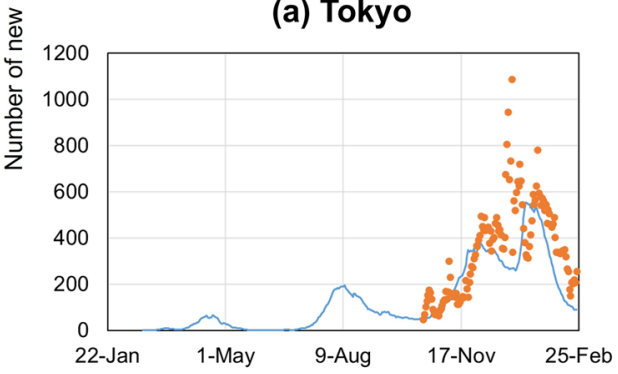

(b) Osaka

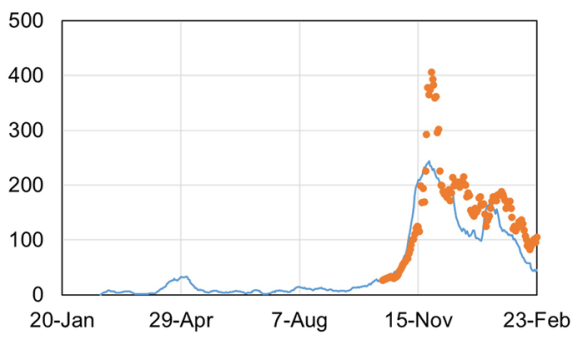

(c) Hokkaido

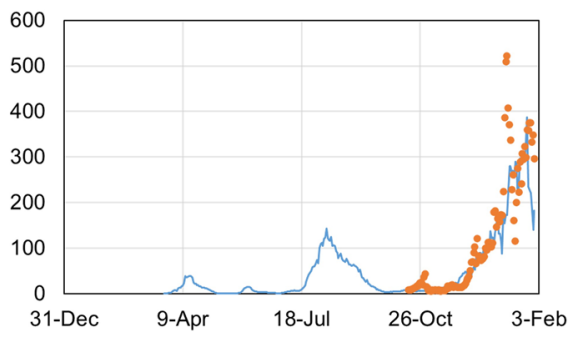

(d) Fukuoka

Figure 3. Forecast results of the number of COVID-19 cases in four prefectures in Japan: (a) Tokyo; (b) Osaka; (c) Hokkaido; and (d) Fukuoka. The locations of the prefectures are shown in the map at the left. These four prefectures were selected because of geographical differences, their large populations, and the high number of COVID-19 cases.

\section{Discussion}

Predictions of COVID-19 cases were made with fewer uncertain assumptions of our model compared with other models. The previous models have been used for long-term predictions by assuming some changes in aspects concerning people's behaviour (e.g., effective reproduction number). However, predicting behaviour change is a challenge in itself, and it is difficult to determine a specific policy in advance based on previous research. By contrast, our model makes no assumptions about behaviour change and region specific information, which increases the reliability of the prediction. This ability to predict the number of cases with less uncertainty will help policymakers develop and initiate policies based on the prediction results. The uncertainty in our model is derived from the accuracy of the model itself, whereas the previous approaches include other uncertainties derived from people's behavioural patterns.

The ability of the model to learn from the real-time data contributes to its flexibility, which is why it could be successfully applied to data in Japan and four individual prefectures of varying population density and geographical conditions. As an additional benefit, the model can keep learning new behaviour and mobility patterns under varying conditions, e.g., increased immunity due to vaccination rates, and spread of new variants, and reflect it on further predictions of infection rates without setting extra assumptions.

Our results suggest that mobility-related policies can show an impact with a delay of 5-10 days in terms of infection rates. The impact is also shown in the period of the second state of emergency in Japan. The second state of emergency was declared on 8 January 2021, and a large drop in infection rates was observed after 17 January 2021 (Supplementary Fig. S1c). A delay of 5-10 days could be costly if it coincides with an exponential increase in cases. Therefore, the ability of the model to predict the beginning of the exponential rise, combined with swift measures affecting targeted mobility profiles while taking into account changes in environmental conditions, could help suppress the resulting peak at minimal costs to society.

The analysis of past mobility data can inform future policies in Japan. Within the training set, changes in mobility patterns occurred at the beginning of August and the middle of September 2020 (Supplementary Fig. S2), with trends in the residential, park, and workplace mobility categories changing significantly. However, the number of cases increased exponentially in August while it was almost steady in September (Fig. 2). To our analysis, a critical difference between the two periods was the length of the holiday period and the outside weather elements. Extremely hot weather in August combined with longer holiday periods resulted in an increase mainly in indoor activities such as residential, grocery and pharmacy. While in September, the milder weather resulted in an increase in outdoor activities, such as shown by the park mobility factors. The cherry blossom season, which usually takes place between March to April, can incur higher level of outdoor activities, e.g., park mobility, similar to that in September, but over a longer duration.

In November, there was a slight improvement in the number of cases without further active intervention from the government (Fig. 2). The improvement in the rate of increase in case numbers happened following a long weekend holiday in mid-November. During this period, workplace mobility dropped and was accompanied by the milder weather in the period of mid to late November, which could have added to the positive effects observed (Supplementary Fig. S2). The government can carefully monitor mobility and environmental factors and predict future trends in the number of cases. 
A gap was observed between the model predictions and actual cases at the end of November to December. During this period, the trends of feature importance within the random forest model shifted (Supplementary Fig. S5), indicating a change in behaviour and resulting in a decrease in prediction accuracy as the model learned new behaviour patterns.

The results obtained for different prefectures reflect the general weather conditions and population behaviour observed in each. For example, the regions of Hokkaido and Fukuoka are less dependent on public transit than in Tokyo and Osaka ${ }^{35}$. The climate in Hokkaido is much colder in winter with milder summer temperatures, which explains the strong correlation between park mobility and temperature in Hokkaido (Supplementary Fig. S6). Generally, Fukuoka experiences an increased number of tourists in summer ${ }^{36}$. In Fukuoka, an increase in infection numbers is observed in June and July (Fig. 3), which could be translated from the increase in transit and recreational mobility (Supplementary Fig. S6). The trends in big cities like Tokyo and Osaka are similar to those observed in the analysis for the whole of Japan. It should be noted that overall the infection rates $\beta(t)$ (specific values already divided by population) appears to be mostly similar between the prefectures starting from November, which implies that population density was not a strong factor in the considered period (from November to February) (Supplementary Fig. S7). There are differences observed in earlier infection rates until August, but those can be attributed to differences in test availability between larger and smaller cities back in that period. Therefore, different weather-specific measures should be taken for different prefectures depending on the observed trends. For example, more indoor restrictions should be taken in Hokkaido in winter, while stricter recreation restrictions to regions similar to Fukuoka should be taken in summer.

The next turning point in Japan is long holidays at the beginning of May (Golden Week). In addition to increased mobility in parks, during Golden Week, people have the opportunity to stay indoors such as in residential areas. Therefore, mobility during Golden Week would be similar to that of August for the whole of Japan. Analysis of the past data thus shows that stricter measures, e.g., restricting residential visits in addition to ongoing restrictions of retail and recreation activities, could be needed before Golden Week to avoid another drastic surge in cases. In reality, a third state of emergency was declared on 25 April 2021.

In general, our model can also work as a warning by combining the $t_{\text {delay }}+1$ day-ahead forecast with the analysis result of individual mobility and environmental trends, which can help the government determine if the upcoming rise in new infections is alarming or not. A forecast of rising case numbers should be combined with a deeper analysis of mobility and environmental trends to determine disturbing combinations of impact factors in addition to the expected magnitude of the rise. Trends similar to those observed in September and November could indicate a slower rise in cases and require mild directed intervention, while trends similar to those observed around the new year in December could be more alarming and strict measures should be applied to avoid a distressing surge. Our model can thus support proactive policy-making depending on the scale of the potential increase in the number of new cases and surrounding mitigating environmental factors.

To increase the versatility of the model, the effect of extrapolation beyond the values in the training set on accuracy needs further verification. For example, the shift in combinations of mobility and weather (e.g., with the Olympic games taking place in summer) from those adopted since February 2020 should be noted. The most robust approach is to wait until the data for the comprehensive training data are collected. However, this approach is less practical considering the need for a rapid response to the pandemic. Further research is needed to determine how much training data is needed for reliable model applications.

Two other aspects of the model could be examined to improve its accuracy. First, regarding forecasts for individual prefectures, there may have been a distance between where people were exposed to the virus and where they tested positive because the model did not consider the possible movement of people between prefectures. Second, regarding forecasts for the whole of Japan, the number of tests might have influenced the measured positive cases. Two consecutive holiday periods occurred in January: Long holidays around the new year and a shorter one in the middle of January. The number of conducted PCR tests fluctuated heavily in these periods compared to the normal pace of testing. This may have led to a smaller number of positive detections. The model results showed a higher expected infection rate than the actual around the new year, indicating a higher underlying infection rate despite the smaller test numbers. However, as the data from the new year period was added to the training set, the model compensated for the gap between actual and predicted values, leading to sharp fluctuations in the prediction of cases between the two holiday periods. The number of PCR tests is thus an important sensitive parameter that should be carefully considered in the analysis of the model results and by the policymakers.

For further applications of our model, extension to different regions and populations will be helpful for regional policy-making as we found that our model could be applied both nationally and for specific prefectures. In addition, further analysis of the calculations about mobility patterns, temperature, and humidity, such as feature importance and sensitivity of the infection rate, will help to identify those factors that contribute to the epidemic, identify the mechanisms of spread within each region, and support more targeted and effective policymaking. By updating the SEIR model, the model may be applicable to countries where vaccination has started ${ }^{14}$, and it may be linked to economic aspects through mobility. Analysis of the economic impact of mobility and combining this information with the prediction model may help to provide precise targeted measures relating to mobility that can balance both the number of cases and economic performance ${ }^{37-41}$. Such information may contribute to the response of each country to the COVID-19 pandemic.

\section{Methods}

The Methods include sections on Datasets and Model of infectious disease epidemics. The Datasets include data on COVID-19 cases, mobility, and environment (temperature and relative humidity). For the Model section, we first explain the basic SEIR model used to calculate the parameters related to epidemics. We next explain our 
method for estimating these parameters based on COVID-19 cases in Japan. Finally, we describe the random forest regression used to estimate the observed infection rate $\beta(t)$.

Datasets. The datasets used in our study included COVID-19 cases $^{26-29}$, mobility $^{30}$, and environment ${ }^{31}$. The details of used data including references are described in the following subsections. We used a simple moving average of the last 7 days to reduce the variability between days of the week. For example, the number of newly infected people on 21 February 2020 was calculated using the data from 15 to 21 February 2020. The data periods used for the analysis are summarized in the Supplementary Table S1. All of the analyses using secondary datasets fulfilled ethical requirements and were performed by following Good Practice of Secondary Data Analysis ${ }^{42}$ and Good Epidemiological Practice ${ }^{43}$.

COVID-19 cases. We used the open data released by the Ministry of Health, Labour and Welfare (MHLW $)^{24}$. The MHLW publishes data about COVID-19 cases for each prefecture and Japan. The number of newly infected people and the cumulative numbers of recovered patients, and COVID-related deaths are available. In this study, four prefectures, Tokyo, Osaka, Hokkaido, and Fukuoka were selected to allow for geographically diversity, while maintaining a high enough number of infections for analysis. The data for Japan were collected directly from the MHLW website ${ }^{26}$. The data for Osaka and Hokkaido were collected from the websites of the respective prefectural governments ${ }^{27,28}$. The data for the other prefectures were collected from the website of Toyo Keizai Online $^{29}$, which provides data for COVID-19 cases for prefectures based on data from the MHLW website ${ }^{26}$.

Mobility. We used the mobility data for Japan released by Google ${ }^{30}$. The data included changes in the following six mobility categories compared to the baseline (median by day of the week for the five weeks from 3 January to 6 February 2020): retail and recreation, parks, transit stations, workplaces, and residential. According to Google, the data show "visitors to (or time spent in) categorized places compared to our baseline days." Here, the baseline day represents the standard value for the corresponding day of the week.

Environment. We used the temperature and relative humidity data released by the Japan Meteorological Agency ${ }^{31}$. The temperature and relative humidity of Tokyo were used for Japan, and the data at the prefectural capital of each prefecture were used as representative data.

Model of infectious disease epidemics. SEIR model. We modelled the spread of COVID-19 using the SEIR model with susceptible $(S)$, exposed $(E)$, infectious $(I)$, and removed $(R)$ compartments ${ }^{6,15}$ for Japan and in individual prefectures (Eq. (1)-(4)). Removed $(R)$ included the recovered and dead population. The SEIR model is an extended version of the SIR mode ${ }^{17}$ to consider incubation periods of infectious diseases. Here, $\alpha$ is the incidence rate, $\beta$ is the infection rate, and $\gamma$ is the removal rate. We considered $\alpha$ and $\gamma$ as disease-specific parameters and assumed that they were constant, whereas we considered $\beta$ as a behaviour- and environmentspecific parameter and assumed that it could change daily. We set the total population $N$ to be constant where $N=S+E+I+R$, and the population of Japan and the four prefectures were used as the values of $N$.

$$
\begin{gathered}
\frac{d S}{d t}=-\beta S I \\
\frac{d E}{d t}=\beta S I-\alpha E \\
\frac{d I}{d t}=\alpha E-\gamma I \\
\frac{d R}{d t}=\gamma I
\end{gathered}
$$

Estimation of parameters. Parameters were estimated based on the data for COVID-19 cases. First, the value of $\gamma$ was estimated by linear regression based on the number of recovered patients and death (see Eq. (4)). For Japan, we used the data from 16 January to 15 October 2020 to calculate $\gamma$, and the same value was used after 15 October 2020. We assumed a constant value of $\gamma$ as it is a disease-specific parameter that does not change significantly with time. For this reason, the obtained $\gamma$ was also used for the calculation for the four prefectures. Second, $E(t)$ was estimated. When the value of $\gamma$ is known, the value of $E(t)$ can be calculated by determining the incidence rate $\alpha$. We used the value of 0.4 for $\alpha$ in accordance to previous research ${ }^{8}$. Finally, the value of $\beta(t)$ for each day was calculated. In the simulation using the SEIR model, we used the value of 0.1 day for the time step $\Delta t$, and the same value of $\beta$ was used within 1 day. The values of $\Delta t$ were varied between 0.01 and 1 day to test the sensitivity of the calculations. The results of the forecasted number of cases shown in Fig. 2(a) were not impacted by the choice of the $\Delta t$ (variations were always smaller than $0.3 \%$ ). The initial conditions set for model parameters were based on the datasets of COVID-19 cases (see Supplementary Table S2). 
Random forest regression. To estimate the value of $\beta(t)$ for mobility, temperature, and relative humidity, we used random forest regression ${ }^{25}$. Random forest regression is a widely-applied machine-learning technique and applicable for describing non-linear relationships. We used scikit-learn in the Python library. A grid search was used with tenfold cross-validation to search for the most accurate hyperparameters. In the first step, an appropriate $t_{\text {delay }}$ from the measurement of input parameters to $\beta(t)$ was calculated as shown in Eq. (5).

$$
\begin{gathered}
\max R^{2} \\
\text { s.t. } \\
t_{\text {delay }} \in Z\left(0 \leq t_{\text {delay }} \leq 19\right) \\
n_{\text {estimators }} \in\{5,10,20,30,50,100,300\} \\
n_{\text {features }} \in N\left(n_{\text {features }} \leq 8\right) \\
n_{\text {depth }} \in\{3,4,5,10,15,20,25,30,40,50,100\} \\
n_{\text {split }} \in\{5,10\}
\end{gathered}
$$

where $n_{\text {estimators [-], }} n_{\text {features }}[-], n_{\text {depth }}[-]$, and $n_{\text {split }}[-]$ represent the number of decision trees, the number of parameters considered in a model, the depth of a model, and the number of samples split, respectively. The results for $R^{2}$ are shown in Supplementary Fig. S1. After fixing $t_{\text {delay }}$ the random forest model was constructed for every day under the condition presented in Eq. (6). To save calculation time in the prediction, hyperparameters (in Eq. (5)) that were never shown as optimal in the grid search during the cross-validation were excluded.

$$
\max R^{2}
$$

$$
\text { s.t. }
$$

$$
\begin{gathered}
n_{\text {estimators }} \in\{5,10,20,30,50,100,300\} \\
n_{\text {features }} \in\{3,4,5,6,7,8\} \\
n_{\text {depth }} \in\{5,10,15,20\} \\
n_{\text {split }}=5
\end{gathered}
$$

\section{Data availability}

Data generated during the study are available in a public repository, https://github.com/SIP-COVID/OpenSource.

\section{Code availability}

The codes used in the work are available at https://github.com/SIP-COVID/OpenSource. Future updates to the data and code will be available at https://www.gsdm-covid-smes.com/.

Received: 26 April 2021; Accepted: 28 July 2021

Published online: 12 August 2021

\section{References}

1. Wu, F. et al. A new coronavirus associated with human respiratory disease in China. Nature 579, 265-269 (2020).

2. Flaxman, S. et al. Estimating the effects of non-pharmaceutical interventions on COVID-19 in Europe. Nature 584, 257-261 (2020).

3. Kuniya, T. Evaluation of the effect of the state of emergency for the first wave of COVID-19 in Japan. Infect. Dis. Model. 5, 580-587 (2020).

4. Chinazzi, M. et al. The effect of travel restrictions on the spread of the 2019 novel coronavirus (COVID-19) outbreak. Science 368, 395-400 (2020).

5. Pei, S., Kandula, S. \& Shaman, J. Differential effects of intervention timing on COVID-19 spread in the United States. Sci. Adv. 6, eabd6370 (2020)

6. Allen, L. J., Brauer, F., Van den Driessche, P. \& Wu, J. Mathematical Epidemiology (Springer, 2008).

7. Cooper, I., Mondal, A. \& Antonopoulos, C. G. A SIR model assumption for the spread of COVID-19 in different communities. Chaos, Solitons Fractals 139, 110057 (2020).

8. Linka, K., Peirlinck, M. \& Kuhl, E. The reproduction number of COVID-19 and its correlation with public health interventions. Comput. Mech. 66, 1035-1050 (2020).

9. Ma, J. Estimating epidemic exponential growth rate and basic reproduction number. Infect. Dis. Model. 5, 129-141 (2020).

10. Chang, S. et al. Mobility network models of COVID-19 explain inequities and inform reopening. Nature 589, 82-87 (2021).

11. Fanelli, D. \& Piazza, F. Analysis and forecast of COVID-19 spreading in China, Italy and France. Chaos Solitons Fractals 134, 109761 (2020).

12. Xu, C., Yu, Y., Chen, Y. Q. \& Lu, Z. Forecast analysis of the epidemics trend of COVID-19 in the USA by a generalized fractionalorder SEIR model. Nonlinear Dyn. 101, 1621-1634 (2020). 
13. Wang, L. et al. An epidemiological forecast model and software assessing interventions on the COVID-19 epidemic in China. J. Data Sci. 18, 409-432 (2021).

14. Jakhar, M., Ahluwalia, P. K. \& Kumar, A. COVID-19 epidemic forecast in different states of India using SIR model. medRxiv https:// doi.org/10.1101/2020.05.14.20101725 (2020).

15. Bubar, K. M. et al. Model-informed COVID-19 vaccine prioritization strategies by age and serostatus. Science 371, 916-921 (2021).

16. Faranda, D. \& Alberti, T. Modeling the second wave of COVID-19 infections in France and Italy via a stochastic SEIR model. Chaos 30, 111101 (2020).

17. Hethcote, H. W. The mathematics of infectious diseases. SIAM Rev. Soc. Ind. Appl. Math. 42, 599-653 (2000).

18. Kraemer, M. U. G. et al. The effect of human mobility and control measures on the COVID-19 epidemic in China. Science 368, 493-497 (2020).

19. Wu, Y. et al. Effects of temperature and humidity on the daily new cases and new deaths of COVID-19 in 166 countries. Sci. Total Environ. 729, 139051 (2020).

20. Ma, Y. et al. Effects of temperature variation and humidity on the death of COVID-19 in Wuhan, China. Sci. Total Environ. 724, $138226(2020)$.

21. Oliveiros, B., Caramelo, L., Ferreira, N. C. \& Caramelo, F. Role of temperature and humidity in the modulation of the doubling time of COVID-19 cases. medRxiv https://doi.org/10.1101/2020.03.05.20031872 (2020).

22. Kuo, C. P. \& Fu, J. S. Evaluating the impact of mobility on COVID-19 pandemic with machine learning hybrid predictions. Sci. Total Environ. 758, 144151 (2021).

23. Rahman, M. M. et al. Machine learning on the COVID-19 pandemic, human mobility and air quality: A review. IEEE Access 9 , 72420-72450 (2021).

24. Roberts, M. et al. Common pitfalls and recommendations for using machine learning to detect and prognosticate for COVID-19 using chest radiographs and CT scans. Nat. Mach. Intell. 3, 199-217 (2021).

25. Breiman, L. Random forests. Mach. Learn. 45, 5-32 (2001).

26. Ministry of Health, Labour and Welfare. Open Data. https://www.mhlw.go.jp/stf/covid-19/open-data.html (2021).

27. Osaka Prefecture. Latest Infection Trend in Osaka Prefecture. https://covid19-osaka.info/ (2021).

28. Hokkaido. COVID-19 Data. https://www.harp.lg.jp/opendata/dataset/1369.html (2021).

29. Toyo Keizai Online. Coronavirus Disease (COVID-19) Situation Report in Japan. https://toyokeizai.net/sp/visual/tko/covid19/ (2021).

30. Google. COVID-19 Community Mobility Reports. https://google.com/covid19/mobility/ (2021).

31. Japan Meteorological Agency. Past Weather Data Download. https://www.data.jma.go.jp/gmd/risk/obsdl/ (2021).

32. World Health Organization. https://www.who.int/emergencies/diseases/novel-coronavirus-2019/question-and-answers-hub/qa-detail/coronavirus-disease-covid-19 (2020).

33. Yu, P., Zhu, J., Zhang, Z. \& Han, Y. A familial cluster of infection associated with the 2019 novel coronavirus indicating possible person-to-person transmission during the incubation period. J. Infect. Dis 221, 1757-1761 (2020).

34. Lauer, S. A. et al. The incubation period of coronavirus disease 2019 (COVID-19) from publicly reported confirmed cases: Estimation and application. Ann. Intern. Med. 172, 577-582 (2020).

35. Ministry of Internal Affairs and Communications. 2010 Population Census. http://www.stat.go.jp/data/kokusei/2010/kihon4/pdf/ gaiyou.pdf (2012).

36. Ministry of Land, Infrastructure, Transport and Tourism. Travel/tourism Consumption Trend Survey. https://www.mlit.go.jp/kanko cho/siryou/toukei/shouhidoukou.html (2017-2020).

37. Silva, P. C. L. et al. COVID-ABS: An agent-based model of COVID-19 epidemic to simulate health and economic effects of social distancing interventions. Chaos Solitons Fractals 139, 110088 (2020).

38. Center for International Development at Harvard University. Horrible Trade-Offs in a Pandemic: Lockdowns, Transfers, Fiscal Space, and Compliance. (Harvard Kennedy School, 2020). https://www.hks.harvard.edu/centers/cid/publications/faculty-worki ng-papers/trade-offs-in-a-pandemic-lockdown.

39. Sampi, J. \& Jooste, C. Nowcasting economic activity in times of COVID-19: An approximation from the Google community mobility report (World Bank, 2020).

40. Mandel, A. \& Veetil, V. The economic cost of COVID lockdowns: An out-of-equilibrium analysis. Econ. Disasters Clim. Chang. 4, 431-451 (2020).

41. Inoue, H. \& Todo, Y. The propagation of economic impacts through supply chains: The case of a mega-city lockdown to prevent the spread of COVID-19. PLoS ONE 15, e0239251 (2020).

42. Swart, E. et al. Good Practice of Secondary Data Analysis (GPS): Guidelines and recommendations. Gesundheitswesen 77, 120-126 (2015).

43. Hoffmann, W. et al. Guidelines and recommendations for ensuring Good Epidemiological Practice (GEP): A guideline developed by the German Society for Epidemiology. Eur. J. Epidemiol. 34, 301-317 (2019).

\section{Acknowledgements}

We thank the Leading Graduate Schools Program, "Global Leader Program for Social Design and Management," by the Ministry of Education, Culture, Sports, Science and Technology for the financial support. We thank Dr. Kevin Montagne and Prof. Hisashi Yoshikawa at The University of Tokyo for consulting project administrations. H.S. is thankful financial support by a Grant-in-Aid for Scientific Research (B) No. 21H01699 from the Japan Society for the Promotion of Science.

\section{Author contributions}

K.M., K.O., and J.K. contributed equally. These authors were jointly involved in the development of the models and codes, design of the study, analysis of the results, and writing of the manuscript. S.B. and H.S. advised on developing research theory and revised the manuscript.

\section{Competing interests}

The authors declare no competing interests.

\section{Additional information}

Supplementary Information The online version contains supplementary material available at https://doi.org/ 10.1038/s41598-021-95617-z.

Correspondence and requests for materials should be addressed to J.K.

Reprints and permissions information is available at www.nature.com/reprints. 
Publisher's note Springer Nature remains neutral with regard to jurisdictional claims in published maps and institutional affiliations.

(c) (i) Open Access This article is licensed under a Creative Commons Attribution 4.0 International License, which permits use, sharing, adaptation, distribution and reproduction in any medium or format, as long as you give appropriate credit to the original author(s) and the source, provide a link to the Creative Commons licence, and indicate if changes were made. The images or other third party material in this article are included in the article's Creative Commons licence, unless indicated otherwise in a credit line to the material. If material is not included in the article's Creative Commons licence and your intended use is not permitted by statutory regulation or exceeds the permitted use, you will need to obtain permission directly from the copyright holder. To view a copy of this licence, visit http://creativecommons.org/licenses/by/4.0/.

(C) The Author(s) 2021 